\title{
AVALIAÇÃO PROSPECTIVA DOS PACIENTES SUBMETIDOS À ARTROPLASTIA TOTAL DO JOELHO COM E SEM COLOCAÇÃO DE DRENO DE SUCÇÃO
}

\author{
SIX MONTH FOLLOW-UP OF PATIENTS SUBMITTED TO TOTAL KNEE \\ ARTHROPLASTY WITH AND WITHOUT PLACEMENT OF SUCTION DRAINAGE DEVICES
}

Marco Antônio Percope de Andrade', Túlio Vinícius de Oliveira Campos², Bruno Flúvio Alves Silva², Mauricio Ernesto de Assis², Lucas de Castro Boechat² ${ }^{2}$ Lúcio Flávio Biondi³ ${ }^{3}$, Wagner Guimarães Lemos ${ }^{3}$, Guilherme Moreira Abreu Silva ${ }^{3}$

\section{RESUMO}

Objetivo: O objetivo deste trabalho é avaliar prospectivamente o padrão de evolução e complicações pós-operatórias relacionadas ao uso de drenos de sucção quando comparado à não utilização destes dispositivos na ATJ. Métodos: Foram incluídos 42 pacientes de um serviço de referência em cirurgia do joelho. Quinze pacientes não receberam e 27 receberam dreno de sucção no pós-operatório. Os parâmetros avaliados foram: amplitude de movimento (ADM), índices hematimétricos, circunferência do joelho e taxa de complicações. O período de observação estendeu-se até o sexto mês de pós-operatório. Resultados: Não foi observada diferença estatisticamente significativa entre os grupos quanto à circunferência do joelho, hemoglobina, hematócrito, taxa de transfusão e índice de infecção. Com relação à amplitude de movimentos, não houve diferença estatística entre os grupos com e sem dreno no pré-operatório $(\mathrm{p}=0,126)$, primeiro DPO $(p=0,583)$, quinto ao sétimo DPO $(p=0,076)$ e seis meses de pós-operatório $(\mathrm{p}=0,848)$. Foi identificada diferença estatisticamente significante entre os grupos na avaliação entre o $14^{\circ}$ e $28^{\circ}$ DPO (p = 0,025). Conclusão: Este estudo conclui que não existe benefício no uso de dreno de sucção fechado na ATJ além de seis meses de pós-operatório. No entanto, a ADM ao final do primeiro mês é melhor no grupo que utilizou o dreno de sucção.

Descritores - Artroplastia do Joelho; Canal de Drenagem; Hemorragia. Amplitude de Movimento Articular

\section{ABSTRACT}

Objective: Our aim is to prospectively evaluate the standard evolution and post-operative complications related to the use of suction drainage devices when compared to not using these devices in TKA. Methods: Forty-two patients from a clinic referred to knee surgery were included. Fifteen patients did not receive suction drainage postoperatively and 27 received suction drainage. The parameters evaluated were the range of movement, hematometric indices, knee circumference, and complications for each group. Patients were observed for six months after the surgical procedure. Results: There were no statistically significant differences in knee circumference, hemoglobin, hematocrit, transfusion rate, and infection index. The analysis of the range of movement did not reveal statistically significant differences between the groups preoperatively ( $p=0.126)$, during the first postoperative day ( $p=0.583$ ), fifth to seventh postoperative day $(p=0.076)$ and at six months followup ( $p=0.848)$. There was a statistically significant difference between groups in the comparison during the 14th and 28th postoperative days $(p=0.025)$. Conclusion: In conclusion, there is no benefit to using closed suction drains beyond six months after TKA. However, the range of movement at the end of the first month is superior in patients that received suction drainage.

Keywords - Arthroplasty, Replacement, Knee. Flood-Bypass Channel. Hemorrhage. Range of Motion, Articular

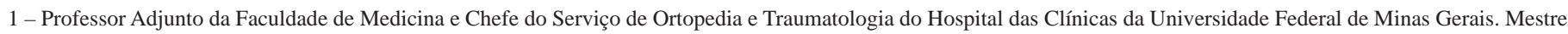
e Doutor em Medicina pela Universidade Federal de São Paulo.

2 - Médico Residente do Serviço de Ortopedia e Traumatologia do Hospital das Clínicas da Universidade Federal de Minas Gerais.

3 - Médico Ortopedista e Preceptor do Grupo de Cirurgia do Joelho do Hospital das Clínicas da Universidade Federal de Minas Gerais.
}

Trabalho realizado no Hospital das Clínicas da Universidade Federal de Minas Gerais.

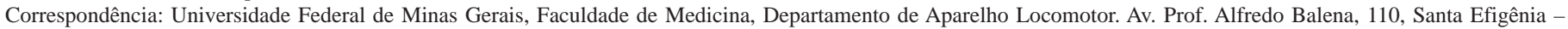
30130-100 - Belo Horizonte/ MG - Brasil. E-mail: mapa.bhz@terra.com.br

Trabalho recebido para publicação: 23/02/2010 , aceito para publicação: 20/08/2010. 


\section{INTRODUÇÃO}

A artroplastia total de joelho (ATJ) é uma das cirurgias ortopédicas mais realizadas no mundo. O sangramento é um evento frequente que se origina principalmente no osso medular e ocorre principalmente após a liberação do torniquete ${ }^{(1)}$.

Desde 1961, após o trabalho de Waugh e Stinchfield ${ }^{(2)}$, os drenos de sucção são empregados em cirurgias ortopédicas $^{(3,4)}$ com a proposta de diminuir formação de hematomas e o risco de infecção.

O hematoma formado prejudica a capacidade de cicatrização devido ao aumento de pressão no sítio cirúrgico e consequente redução da perfusão tecidual, apresenta diminuída capacidade de opsonizar bactérias e é considerado fator predisponente ao aparecimento de infecções ${ }^{(5)}$. Além disso, considera-se que a mobilização precoce seja mais difícil e a dor no pós-operatório maior quando hematomas são formados ${ }^{(6-8)}$, com risco aumentado de deiscência da ferida e formação de fibrose, levando à rigidez $\operatorname{articular}^{(9,10)}$.

Em contrapartida, os drenos podem funcionar como fonte de contaminação ${ }^{(5,9,11)}$ e impedir o efeito de tamponamento natural que ocorre quando as feridas cirúrgicas são simplesmente fechadas por sutura. Por isso, a perda sanguínea poderia ser maior quando drenos são utilizados $^{(7)}$.

O objetivo do presente estudo é avaliar prospectivamente o padrão de evolução e complicações pósoperatórias relacionadas ao uso de drenos de sucção quando comparado à não utilização destes dispositivos na ATJ.

\section{MATERIAIS E MÉTODOS}

Foram estudados 42 pacientes portadores de gonartrose primária ou secundária. Os pacientes foram operados no período de outubro de 2007 a abril de 2009. Quinze pacientes foram incluídos no grupo que não receberia drenos e 27 pacientes receberam drenos.

Os pacientes foram randomizados logo antes do fechamento da ferida operatória após hemostasia com eletrocautério. A definição do grupo era feita a partir de sorteio com moeda em que "cara” indicava o grupo com dreno e "coroa" o grupo sem dreno.

Todos os pacientes assinaram termo de consentimento após esclarecimento pelo médico assistente. Foram excluídos pacientes deficientes mentais, portadores de distúrbios de coagulação ou aqueles que não concordaram com a participação no estudo.
O pré-operatório consistia na coleta de sangue para reserva de hemoderivados, hemograma e coagulograma além de avaliação de risco cirúrgico pela equipe de anestesiologia. Foi também mensurada a amplitude de movimento do joelho a ser operado (ADM) utilizando goniômetro e a circunferência articular tomando como referência o 1/3 médio da patela. Estes mesmos princípios foram utilizados na avaliação pós-operatória. A coleta de dados foi realizada por médicos do serviço de ortopedia previamente treinados (TVOC, LCB, MEA).

Todos os pacientes receberam profilaxia para eventos tromboembólicos com heparina não fracionada (5.000UI SC oito/oito horas) iniciada no dia da cirurgia, e profilaxia para infecção do sítio cirúrgico com cefazolina (EV de oito/oito horas) com dose ajustada para o peso do paciente por um período de 24 horas. Todos os procedimentos cirúrgicos foram realizados por cirurgiões ortopédicos membros do serviço de cirurgia do joelho. O torniquete pneumático com pressão ajustada de acordo com a pressão arterial sistólica do paciente foi utilizado durante o procedimento pelo período máximo de 120 minutos. Os pacientes receberam uma prótese de joelho Nex Gen cimentada (Zimmer, Inc. Warsaw, IN). O treino de marcha com andador foi iniciado no primeiro ou segundo dia de pós-operatório (DPO) de acordo com tolerância do paciente.

Os pacientes sorteados para o grupo com dreno receberam um dreno Portovac (Simon, número 3,2) que foi mantido por um período de 24 horas.

A avaliação do sangramento foi realizada a partir dos valores médios da concentração de hemoglobina e hematócrito no primeiro DPO em comparação aos valores prévios. Foi mensurado, também, o volume de sangue drenado nas primeiras 12 e 24 horas nos pacientes que receberam dreno. Esses parâmetros foram avaliados em conjunto com as transfusões recebidas pelos respectivos pacientes. O critério para transfusão foi a presença de sinais e sintomas clínicos que pudessem ser explicados por anemia.

A avaliação da amplitude de movimento foi feita no primeiro, sétimo, entre o $14^{\circ}$ e $28^{\circ}$ dia de pós-operatório e aos seis meses de cirurgia. A circunferência articular foi mensurada no primeiro, sétimo e no intervalo entre o $14^{\circ}$ e o $28^{\circ}$ dia de pós-operatório.

Foram anotadas as intercorrências ocorridas durante a internação e no seguimento pós-operatório.

Os dados foram analisados através do programa SPSS for Windows versão 10.0.1. As variáveis numéricas contínuas tiveram sua normalidade testada e de 
acordo com o resultado foi proposto o teste estatístico. Variáveis com distribuição normal foram comparadas pelo teste $t$ de Student e ANOVA. Variáveis com distribuição não normais foram comparadas utilizando-se o teste de Kruskal-Wallis.

\section{RESULTADOS}

Os pacientes foram avaliados pelo período de seis meses com consultas periódicas, conforme protocolo descrito. Uma paciente não foi avaliada após o terceiro DPO, pois faleceu com quadro sugestivo de tromboembolismo pulmonar.

As médias de idade, permanência hospitalar, estatura, peso, duração do procedimento cirúrgico, hemoglobina/ hematócrito, circunferência do joelho e amplitude de movimento no pré e pós-operatório estão registradas na Tabela 1.

A comparação da média da circunferência do joelho no pré-operatório ( $\mathrm{p}=0,962)$, no pós-operatório imediato $(\mathrm{p}=0,900)$ e no quinto ao sétimo DPO $(\mathrm{p}=0,931)$ não revelou diferença significativa entre os grupos.

Com relação à amplitude de movimentos, não houve diferença estatística entre os grupos com e sem dreno no pré-operatório ( $\mathrm{p}=0,126)$, primeiro DPO $(\mathrm{p}=0,583)$, quinto a sétimo DPO ( $\mathrm{p}=0,076)$ e seis meses de pósoperatório ( $p=0,848)$. Foi identificada diferença estatisticamente significante entre os grupos na avaliação entre o $14^{\circ}$ e $28^{\circ} \mathrm{DPO}(\mathrm{p}=0,025)$. Nesse caso, os pacientes do grupo que recebeu dreno de sucção no pós-operatório apresentaram ADM maior que os do grupo sem dreno. Vale ressaltar que essa diferença começa a se mostrar no sétimo DPO quando existe uma tendência a significância ( $p=0,076)$, com média de ADM maior no grupo de pacientes que recebeu dreno (Figura 1).

Na avaliação indireta das perdas sanguíneas, não se observou diferença estatística na média de hemoglobina e hematócrito do pré e pós-operatório entre os grupos. Evidentemente, a comparação entre a hemoglobina média pré e pós-operatória geral e para cada grupo mostrou diferença significativa ( $\mathrm{P}<0,000$ para todas as comparações). Dois pacientes de cada grupo receberam transfusão, o que representa $7,4 \%$ dos pacientes do grupo com dreno e $13,3 \%$ dos pacientes sem dreno.

$\mathrm{O}$ volume drenado, nos pacientes que receberam drenos de sucção contínua, concentrou-se nas primeiras 12 horas (Figura 2).

Tabela 1 - Dados colhidos no pré e pós-operatório de pacientes submetidos à artroplastia total do joelho com e sem a utilização de dreno.

\begin{tabular}{|c|c|c|c|}
\hline & Sem dreno & Com dreno & Valor $p$ \\
\hline Número de pacientes & 15 & 27 & - \\
\hline Idade (anos) & $69,93 \pm 7,11$ & $69,00 \pm 9,31$ & 0,738 \\
\hline Permanência hospitalar (dias) & $2,31 \pm 0,67$ & $2,14 \pm 0,35$ & 1 \\
\hline Estatura (m) & $1,68 \pm 0,06$ & $1,61 \pm 0,08$ & 0,131 \\
\hline Peso (kg) & $74,2 \pm 11,76$ & $74,6 \pm 14,04$ & 0,711 \\
\hline Duração (minutos) & $152,78 \pm 37,43$ & $137,62 \pm 19,47$ & 0,49 \\
\hline Hemoglobina pré-operatória (g/dL) & $14,18 \pm 2,04$ & $14,12 \pm 1,54$ & 0,914 \\
\hline Hematócrito pré-operatório (g/dL) & $41,07 \pm 4,88$ & $42,06 \pm 3,83$ & 0,421 \\
\hline Hemoglobina pós-operatória & $10,47 \pm 1,74$ & $10,12 \pm 1,14$ & 0,429 \\
\hline Hematócrito pós-operatório & $30,30 \pm 4,26$ & $30,60 \pm 3,33$ & 0,757 \\
\hline Circunferência pré-operatória $(\mathrm{cm})$ & $40,27 \pm 3,55$ & $40,35 \pm 4,66$ & 0,962 \\
\hline Circunferência pós-operatória $(\mathrm{cm})$ & $43,05 \pm 3,99$ & $43,27 \pm 4,67$ & 0,9 \\
\hline $\begin{array}{c}\text { Circunferência pós-operatória } \\
\text { quinto-sétimo DPO }(\mathrm{cm})\end{array}$ & $43,5 \pm 4,94$ & $42,09 \pm 4,58$ & 0,931 \\
\hline ADM pré-operatória & $109,26 \pm 19,99$ & $98,33 \pm 24,54$ & 0,126 \\
\hline ADM primeiro DPO & $65,67 \pm 18,21$ & $69,81 \pm 25,36$ & 0,583 \\
\hline ADM sétimo DPO & $73,85 \pm 20,63$ & $85,96 \pm 18,97$ & 0,076 \\
\hline ADM $14-28^{\circ} \mathrm{DPO}$ & $78,64 \pm 25,99$ & $95,20 \pm 14,61$ & $0,025^{*}$ \\
\hline ADM seis meses & $109,00 \pm 15,17$ & $107,08 \pm 15,73$ & 0,848 \\
\hline
\end{tabular}

Variáveis contínuas expressas em média \pm desvio padrão. ADM: amplitude de movimento mensurada por goniometria. DPO: dia pós-operatório * $\mathrm{p}<0,05$ 


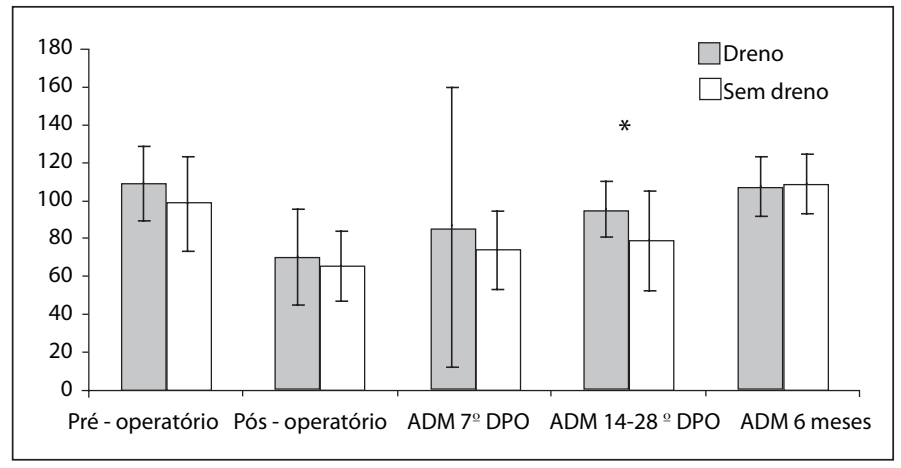

Figura 1- Média e desvio padrão da ADM em graus de pacientes submetidos à artroplastia total do joelho com e sem a utilização de dreno no pré-operatório e até o sexto mês pós-operatório.

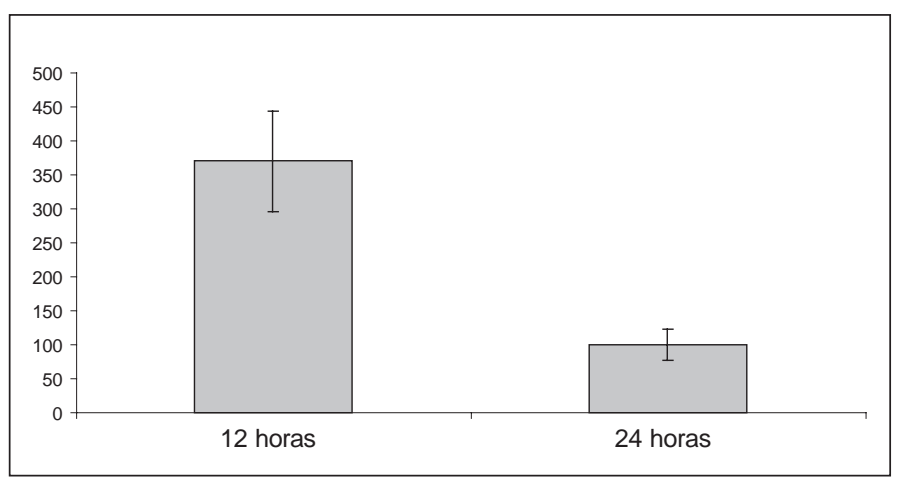

Figura 2 - Média e desvio padrão da drenagem $(\mathrm{mL})$ nos pacientes que receberam drenos de sucção no período pós-operatório.

Com relação às complicações pós-operatórias, no grupo de pacientes em que se utilizou dreno houve três casos considerados graves (deiscência de ferida na primeira semana de pós-operatório, infecção profunda com necessidade de retirada da prótese e limitação da flexão). No grupo de pacientes que não receberam dreno houve um óbito com suspeita de tromboembolismo pulmonar e um processo infeccioso profundo que evoluiu para retirada da prótese.

\section{DISCUSSÃO}

O uso de drenos em artroplastias de joelho é tema controverso $^{(3,4,7,8,10,12-19)}$. Inúmeros trabalhos têm investigado o papel dos drenos de sucção na prevenção de complicações pós-operatórias em cirurgias ortopédicas, mas até o momento poucas conclusões foram ob tidas $^{(2-4,6,10,12-14,16-18,20,21)}$. Está estabelecido que na ATJ cimentada o risco de hemorragias encontra-se diminuído em comparação às não cimentadas e, por isso, questiona-se a necessidade de implantação de drenos naqueles $\operatorname{casos}^{(13)}$.

Este trabalho mostra que a utilização de dreno não modifica a evolução dos pacientes com relação aos índi- ces hematimétricos. Esses dados se encontram de acordo com os de Kim et $a l^{(15)}$, Esler et $a l^{(7)}$, Adalberth et $a l^{(10)}$, Ritter et $a l^{(3)}$, Beer et $a l^{(14)}$, Jenny et $a l^{(17)}$, Niskanen et $a l^{(16)}$ que não perceberam diferença nos parâmetros analisados entre grupos tratados com e sem dreno em seus trabalhos prospectivos. Conceito contrário foi defendido por Leb et al, Waugh e Stinchfieldl ${ }^{(2)}$ e Simpson et al apud Adalberth et al(10) que afirmaram sobre a utilidade de drenos de sucção em cirurgia ortopédica.

Leb et $a l^{(22)}$ usaram o hematócrito como parâmetro para estimar a perda sanguínea, semelhante à metodologia utilizada neste estudo, que utilizou os níveis de hemoglobina e o hematócrito para a avaliação da perda sanguínea.

Porteus e Bartlett ${ }^{(18)}$, tomando como parâmetro o sangramento acima de $1.000 \mathrm{ml}$ em próteses não cimentadas e hibridas de joelho, mantêm a indicação do uso de drenos nestas situações. O presente estudo não demonstrou nenhum caso com drenagem igual ou superior a esse volume tão expressivo, devendo-se considerar, no entanto que todas as próteses foram cimentadas.

Uma meta-análise ${ }^{(19)}$ mostrou que a taxa de infecção em artroplastias totais de quadril/joelho que receberam ou não dreno era respectivamente $0,5 \%$ e $0,2 \%$. Também não houve diferença quanto ao índice de reoperação, hematoma, deiscência, aumento de volume do membro, trombose venosa profunda, amplitude de movimento, função, tempo de hospitalização e força. A única vantagem comprovada da utilização do dreno é a redução do vazamento de sangue pela ferida operatória. Esses dados não puderam ser confirmados neste estudo pelo tamanho da casuística e reduzido número de complicações.

Kumar et al $^{(23)}$ monitorizaram a drenagem por dispositivos de sucção contínua e concluiu que a maior parte da drenagem ocorre nas primeiras 12 horas (em média $562 \mathrm{ml}$ nas primeiras 12 horas e $627 \mathrm{ml}$ em 24 horas) e, por isso não haveria vantagem em deixar os dispositivos por tempo maior. Esse mesmo padrão de drenagem foi confirmado nos estudos de Ritter et $a^{(3)}$, Andrade ${ }^{(24)}$, Willemen et $a l^{(12)}$, Niskanen et $a^{(16)}$ e no presente trabalho, o que despertou o interesse em um futuro estudo comparando-se a utilização de drenos por 12 e 24 horas.

A comparação da evolução da amplitude de movimentos nos dois grupos mostrou diferença estatisticamente significante na avaliação do $14^{\circ}$ ao $28^{\circ}$ DPO. Entretanto, na avaliação de seis meses, não houve diferença estatisticamente significante. Esse achado está de acordo com trabalhos de alguns autores que contestam 
a necessidade do dreno para um melhor resultado de ADM no longo prazo $^{(3,4,7,8,10,12-19)}$. A amplitude de movimento precoce maior nos pacientes com dreno pode ser justificada pela formação de hematoma reduzida que ocorre nesses pacientes. A ADM nos primeiros dias de pós-operatório semelhante provavelmente deve-se a um componente de dor equivalente nos dois grupos.

\section{CONCLUSÃO}

Este estudo conclui que não existe benefício no uso de dreno de sucção fechado na ATJ além de seis meses de pós-operatório. No entanto, a ADM ao final do primeiro mês é melhor no grupo que utilizou o dreno de sucção.

\section{REFERÊNCIAS}

1. Burke DW, O'Flynn H. Primary total knee arthroplasty. In: Chapman MW. Chapman's orthopaedic surgery. 3rd edition. Philadelphia: Lippincott Williams \& Wilkins; 2001. p. 2870-95.

2. Waugh TR, Stinchfield FE. Suction drainage of orthopaedic wounds. J Bone Joint Surg Am. 1961;43(7):939-46.

3. Ritter MA, Keating M, Faris M. Closed wound drainage in total hip or total knee replacement. J Bone Joint Surg Am. 1994;76(1):35-8.

4. Ovadia D, Luger E, Bickels J, Menachem A, Dekel S. Efficacy of closed wound drainage after total joint arthroplasty. J Arthroplasty, 1997;12(3):317-21.

5. Alexander JW, Korelitz J, Alexander NS. Prevention of wounds infections. Am J Surg. 1976;132(1):59-63.

6. Bryan RS, Dickson JH, Taylor W. Recovery of the knee following meniscectomy. J Bone Joint Surg Am. 1969;51(5):973-8.

7. Esler, CNA; C. Blakeway C; Fiddian, NJ. The use of a closed-suction drain in total knee arthroplasty. A prospective randomized study. J Bone Joint Surg Br. 2003;85(2):215-7.

8. Parker MJ, Roberts CP, Hay D. Closed suction drainage for hip and knee arthroplasty. A meta-analysis. J Bone Joint Surg Am. 2004;86(6):1146-52.

9. Raves JJ, Slifkin M, Diamond DL. A bacteriologic study comparing closed suction and simple conduit drainage. Am J Surg. 1984;148(5):618-20.

10. Adalberth G, Byström S, Kolstad K, Mallmin H, Milbrink J. Postoperative drainage of knee arthroplasty is not necessary: a randomized study of 90 patients. Acta Orthop Scand. 1998;69(5):475-8.

11. Magee C, Rodeheaver G, Golden GT, Edgerton MT, Edilich RF. Potentiation of wound infection by surgical drains. Am J Surg. 1976;131(5):547-9.

12. Willemen D, Paul J, White SH, Crook DW. Closed suction drainage following knee arthroplasty. Effectiveness and risks. Clin Orthop Relat Res. 1991;(264):232-4.
13. Mylod AG Jr, France MP, Muser DE, Parsons JR. Perioperative blood loss associated with total knee arthroplasty. A comparison of procedures performed with and without cementing. J Bone Joint Surg Am. 1990;72(7):1010-2.

14. Beer KJ, Lombardi AV Jr, Mallory TH, Vaughn BK. The efficacy of suction drains after routine total joint arthroplasty. J Bone Joint Surg Am. 1991;73(4):584-7.

15. Kim YH, Cho SH, Kim RS. Drainage versus nondrainage in simultaneous bilateral total hip arthroplasties. J Arthroplasty. 1998;13(2):156-61.

16. Niskanen RO, Korkala OL, Haapala J, Kuokkanen HO, Kaukonen JP, Salo SA. Drainage is of no use in primary uncomplicated cemented hip and knee arthroplasty for osteoarthritis: a prospective randomized study. J Arthroplasty. 2000;15(5):567-9.

17. Jenny JY, Boeri C, Lafare S. No drainage does not increase complication risk after total knee prosthesis implantation: a prospective, comparative, randomized study. Knee Surg Sports Traumatol Arthrosc. 2001;9(5):299-301.

18. Porteus AJ, Bartlett RJ. Post-operative drainage after cemented, hybrid and uncemented total knee replacement. Knee. 2003;10(4):371-4.

19. Parker MJ, Livingstone V, Clifton R, Mackee A. Closed suction surgical wound drainage after orthopaedic surgery. Cochrane Database Syst Rev. 2007;(3):CD001825.

20. Browett JP, Gibbs AN, Copeland SA, Deliss LJ. The use of suction drainage in the operation of meniscectomy. J Bone Joint Surg Br. 1978;60(4):516-9.

21. Nixon $\mathrm{J}$. Wound drainage the long term results after primary hip and knee arthroplasty. J Bone Joint Surg. 2000;82(Suppl 2):125.

22. Leb RB, Parker RD, Cohn BT, Fabian V. The efficacy of closed suction drainage in total knee arthroplasty. Orthop Trans. 1995;19:332-3.

23. Kumar S, Penematsa S, Parekh S. Are drains required following a routine primary total joint arthroplasty? Int Orthop. 2007;31(5):593-6.

24. Andrade MAP. Drenagem pos-operatória após artroplastia total do joelho. Rev Bras Ortop. 1998;33(8):659-61. 